\title{
Remarks on the Ural spider fauna (Arachnida: Aranei), 14. On the spider fauna of the Kytlym plexus of mountains (the North Urals)
}

\author{
Заметки по фауне пауков Урала (Arachnida: Aranei), 14. О фауне \\ пауков КытАымского горного узма (Северный Урал)
}

\author{
S.L. Esyunin*, A.I. Ermakov**, Yu.E. Mikhailov*** \\ С.А. Есюнин*, А.И. Ермаков**, Ю.Е. Михаймов***
}

\footnotetext{
* Perm State University, Bukireva street 15, Perm 614990, Russia. E-mail: Sergei.Esyunin@psu.ru

** Institute of Plant and Animal Ecology of the RAS, 8 March street 202, Ekaterinburg 620114, Russia. E-mail: ermakov@ipae.uran.ru

*** Urals State Forestry-Engineering University, Sibirsky trakt 37, Ekaterinburg 620100, Russia. E-mail: yuemikhailov@gmail.com.

* Пермский государственный национальный исследовательский университет, ул. Букирева 15, Пермь 614990, Россия.

** Институт экологии растений и животных, ул. 8 Марта, 202, Екатеринбург 620144, Россия.

*** Уральский государственный лесотехнический университет, Сибирский тракт, 37, Екатеринбург 620100, Россия.
}

KEY WORDS: spider fauna, the North Urals, Ekaterinburg Area.

КЛЮЧЕВЫЕ СЛОВА: фауна пауков, Северный Урал, Свердловская область.

ABSTRACT. A check-list of 96 spider species from 74 genera and 18 families collected from the vicinity of Kytlym (the North Urals) is presented. The records of Alopecosa aculeata (Clerck, 1757) from the vicinity of Kytlym are shown to be misidentifications, whereas that of Xysticus audax (Schrank, 1803) remains doubtful.

РЕЗЮМЕ. Для окрестностей пос. Кытлым (северный Урал) приведен список из 96 видов из 74 родов 18 семейств пауков. Указания для окрестностей пос. Кытлым Alopecosa aculeata (Clerck, 1757) ошибочно, а находка Xysticus audax (Schrank, 1803) сомнительна.

\section{Introduction}

The spider fauna of the eastern macroslope of North Urals remains poorly known [Esyunin, Efimik, 1996, 1997a,b]. D.E. Kharitonov [1926, 1936] described two small spider collections from this region at the beginning of XX century. During the last decade, the mountain fauna of Ekaterinburg (=Sverdlovsk) Area has been surveyed by researchers from the Institute of Plant and Animal Ecology of the RAS. The first results of this survey devoted to the spider fauna of Denezhkin Kamen' Ridge were recently published by Esyunin \& Ermakov [2012], who presented a list of 196 spider species recorded to date from that local fauna.

The second local spider fauna studied in the aforementioned region is that of the mountains surrounding Kytlym settlement. A small collection of 11 spider species collected by A.O. Tauson and K.N. Igoshina was investigated by D.E. Kharitonov [1926]. Further information on the spiders occurring in the vicinity of Kytlym can be found in the following two works: Esy- unin [2006] who reported on five linyphiid species and Phrurolithus festivus (C.L. Koch, 1835), and Tuneva [2007] reported on two Micaria species and on Arboricaria subopaca (Westring, 1861). Thus, by the beginning of our research, 20 spider species had been known from the vicinity of Kytlym. Of them, a record of Alopecosa aculeata (Clerck, 1757) was erroneous and that of Xysticus audax (Schrank, 1803) remains doubtful.

In the present paper, we have summarized both the literature-derived and newly collected data on the spiders from the vicinity of Kytlym. A total of 96 species of 74 genera and 18 families is currently known.

Kytlym settlement (c. 59 $36^{\prime} \mathrm{N}, 59^{\circ} 03^{\prime} \mathrm{E}$, Karpinsk District, Sverdlovsk [=Ekaterinburg] Area) is located on the eastern Ural macroslope, on the border between the Middle and North Urals. The intermountain hollow is surrounded by ridges of average height. Climate is cold and excessively humid. The average temperature of January is $-16.8^{\circ} \mathrm{C}$ and of July $+14.0^{\circ} \mathrm{C}$; annual precipitation is $500-700 \mathrm{~mm}$ in the valleys, and up to $1200 \mathrm{~mm}$ in the mountains. The mountain-forest belt goes up to $900 \mathrm{~m}$ a.s.l. The coniferous taiga-forest consisting mainly of Picea, Abies and Pinus sibirica predominates. Mountain tundra and stone streams (the Siberian term "kurumy") are widespread above $1000 \mathrm{~m}$ a.s.1. [Moiseev et al., 2010].

\section{Material and methods}

This study is based on the spider collections made by A.I. Ermakov in 1996, 1997, 1981 and 2001, Yu.E. Mikhailov in 2011 and the student O.A. Pukalchuk in 2003-2004. The surveyed localities are described below. 
1. Tylaisko-Konzhakovsko-Serebryanskiy Mt. Ridge (extending across $20 \mathrm{~km}$ from the west to the east), 14 15 km N of Kytlym: Garevaya Mt., Tylaiskiy Kamen' Mt., Malyi Konzhakovskiy Kamen' Mt. \& Trapetsiya Mt., VII-VIII.2011, Yu.E. Mikhailov, and Serebryanskiy Kamen' Mt., VII.1996, A.I. Ermakov.

2. Kos'vinskiy Kamen' Mt., c. 7 km W of Kytlym, VII.1996, VII.1997, VI.1981, VIII.2001, A.I. Ermakov; VI.2003, VIII.2005, O.A. Pukalchuk.

3. Vicinity of Kytlym, VI-VII.2003, VI-VII.2004, O.A. Pukalchuk.

4. Mountain Ridge $2-11 \mathrm{~km} \mathrm{SE}$ of Kytlym, extending across $14 \mathrm{~km}$ from the south-west to the north-east: Bugor Mt., Semichelovechiya Mt. \& Kazanskiy Kamen’ Mt., VIII.2001, A.I. Ermakov; Kolpak Mt., VIVII.2003, VI-VII.2004, O.A. Pukalchuk.

The names of species ranges follows Esyunin \& Marusik [2011]. The studied material is shared between the collections of the Institute of Plant and Animal Ecology of the RAS, Ekaterinburg, Russia (IPAE) and the Department of Zoology of the Perm State University, Perm, Russia (PSU). Abbreviations used in the text: AIE - A.I. Ermakov, OAP - O.A. Pukalchuk, YEM - Yu.E. Mikhailov, juv. - juvenile specimens, Mt. - mountain.

\section{ARANEIDAE}

Aculepeira carbonarioides (Keyserling, 1892)

MATERIAL. $6 O^{7} \sigma^{7}, 1$ ㅇ (PSU-16), [1]: Serebryanskiy Kamen' Mt., no habitat data, 28.VII.1925, À. Tauson; 2 우 (IPAE) [1]: Serebryanskiy Kamen' Mt., 1200 m a.s.l., stone streams (=kurumy), 8.VII.1997, AIE; 1 O" $^{7} 6$ 우 (PSU-4786), [2] Kos'vinskiy Kamen' Mt., stone streams (=kurumy), 26.VI.2003, OAP.

Kharitonov [1926: as Araneus (Epeira) hyperboreus Kulczyński, 1908]: [1]: Konzhakovskiy Kamen’ Mt., Serebryanskiy Kamen' Mt., [3].

DISTRIBUTION. NEEuropean-TransNearctic arcto-alpine range.

Araneus marmoreus Clerck, 1757

MATERIAL. 2 우우 (PSU-914), [2], mountain forest, 13.VII.1996, AIE; $10^{7}$ (PSU-4810), [3], on Picea, 7.VII.2003, OAP; 1 + (PSU-4810), [4]: Bugor Mt., mountain forest, 2.VII.2001, AIE.

Kharitonov [1926]: Topol Kamen' Mt. on Kos'va River.

DISTRIBUTION. Circum-Holarctic temperate range.

Araneus quadratus Clerck, 1757

MATERIAL. $10^{\top}, 1$ ㅇ (PSU-4836), [3], Pinus-Picea forest and meadow, VII.2003, OAP.

DISTRIBUTION. West-Central Palaearctic polyzonal range.

Araneus saevus (L. Koch, 1872)

Araneus zimmermanni (Thorell, 1875): Kharitonov, 1926: 50, Fig. 1 ().

Kharitonov [1926: as Araneus zimmermanni (Thorell, 1875)]: Rebro Kamen' Mt. on Kos'va River, 400 m a.s.l.

DISTRIBUTION. Circum-Holarctic temperate range.

Cercidia prominens (Westring, 1851)

MATERIAL. 1 (PSU-4829), [3], meadow, 1.VII.2004, OAP. DISTRIBUTION. Circum-Holarctic temperate range.
Cyclosa conica (Pallas, 1772)

MATERIAL. 3 우 (PSU-4788), [4]: Kolpak Mt., southern slope, forest, 9.VII.2003, OAP

DISTRIBUTION. Circum-Holarctic temperate range.

\section{CLUBIONIDAE}

Clubiona kulczynskii Lessert, 1905

Clubiona uralensis Charitonov, 1926, Fig. 5, p. 52 (†).

MATERIAL. 2 우 (PSU-949), [2], $1500 \mathrm{~m}$ a.s.1., Carex-moss tundra, 9.VII.1996; $1200 \mathrm{~m}$ a.s.1., mountain meadow, 10.VII.1997, AIE; 1 + , [4]: Bugor Mt., tundra, 11.VIII.2001, AIE.

Kharitonov [1926: as Clubiona uralensis]: [3], forest meadow.

DISTRIBUTION. Circum-Holarctic temperate range.

Clubiona subsultans Thorell, 1875

MATERIAL. 2 우 (PSU-2739), [4]: Bugor Mt., Mountain taiga, 2 \& 14.VIII.2001, AIE; 1 + (PSU-2739), [4]: Kazanskiy Kamen' Mt., tundra, sweeping, 3.VIII.2001, AIE.

DISTRIBUTION. Trans-Palaearctic temperate range.

\section{CORINNIDAE}

Phrurolithus festivus (C.L. Koch, 1835)

MATERIAL. 1 + (PSU-1009), [2], $900 \mathrm{~m}$ a.s.1., tundra, 10.VII.1996, AIE

Esyunin [2006]: [2].

DISTRIBUTION. Trans-Palaearctic nemoral range.

\section{DICTYNIDAE}

Dictyna arundinacea (Linnaeus, 1758)

MATERIAL. $1 \mathrm{O}^{7}$ (PSU-4781), [2], no habitat data, 26.VI.2003, OAP; 3 우 (PSU-4781), [3], edge of mixed forest, 17.VI.2003, OAP.

DISTRIBUTION. Circum-Holarctic polyzonal range.

Dictyna pusilla Thorell, 1856

MATERIAL. $10^{7}$ (PSU-4833), [2], no habitat data, 26.VI.2003, OAP; 1 \& (PSU-4833), [3], Betula-Picea forest, 6.VII.2004, OAP.

DISTRIBUTION. Trans-Palaearctic temperate range.

\section{GNAPHOSIDAE}

Arboricaria subopaca (Westring, 1861) OAP.

MATERIAL. 1 + (PSU-4845), [3], mixed forest, 10.VII.2004,

Tuneva [2007]: [3]

DISTRIBUTION. Trans-Palaearctic nemoral range.

Drassodes lapidosus (Walckenaer, 1802)

MATERIAL. 1 + (IPAE), [1]: Serebryanskiy Kamen’ Mt., 1200 $m$ a.s.l., dwarf Betula stand, 12.VII.1996, AIE; 2 O $^{7} \sigma^{7}, 1$ ( (PSU4804), [4]: Kolpak Mt., stone streams (=kurumy), 18.VII.2004, OAP.

DISTRIBUTION. ?Trans-Palaearctic boreo-mountain range.

Drassodes pubescens (Thorell, 1856)

MATERIAL. $10^{\top}$ (PSU-4830), [3], meadow, 11.07.2004, OAP.

DISTRIBUTION. Trans-Palaearctic nemoral range.

Gnaphosa muscorum (L. Koch, 1866)

MATERIAL. $1 \sigma^{7}$ (IPAE), [1]: Tylaiskiy Kamen' Mt., grasslichen-moss tundra, 25-28.VII.2011, YEM.

DISTRIBUTION. Subcircum-Holarctic boreomountain range. 
Haplodrassus signifer (C.L. Koch, 1839)

MATERIAL. 1 \& (PSU-961), [1]: Serebryanskiy Kamen' Mt., $1200 \mathrm{~m}$ a.s.1., dwarf Betula stand, 12.VII.1996, AIE.

DISTRIBUTION. Circum-Holarctic polyzonal range.

Haplodrassus soerenseni (Strand, 1900)

MATERIAL. $10^{7}$ (PSU-963), [1]: Serebryanskiy Kamen' Mt., 1200 m a.s.l., dwarf Betula stand, 12.VII.1996, AIE.

DISTRIBUTION. Trans-EuroSiberian temperate range.

Micaria alpina L. Koch, 1872

MATERIAL. $4 \mathrm{O}^{\top} \mathrm{O}^{\top}, 1$ + (IPAE), [1]: Garevaya Mt., Vaccinium-grass tundra with dwarf Betula stand, 23-27.VII.2011, YEM; $1 \mathrm{O}^{7}$ (PSU-1012), [2], 1200-1300 m a.s.1., mountain meadow, 10.VII.1997, AIE

Tuneva [2007]: [1,2].

DISTRIBUTION. Subcircum-Holarctic boreomountain range.

Micaria silesiaca L. Koch, 1875 OAP.

MATERIAL. 1 \% (PSU-4841), [3], Lobva River bank, VII.2003,

Tuneva [2007]: [3].

DISTRIBUTION. West-Palaearctic temperate range.

Zelotes latreillei (Simon, 1878)

MATERIAL. $1 \sigma^{7}$ (PSU-1040), [1]: Serebryanskiy Kamen' Mt., $1200 \mathrm{~m}$ a.s.1., dwarf Betula stand, 12.VII.1996, AIE; $1 \mathrm{O}^{7}$ (PSU-4806), [4]: Kolpak Mt., stone streams (=kurumy), 18.VII.2004, OAP.

DISTRIBUTION. West-Palaearctic temperate range.

\section{HAHNIIDAE}

Cryphoeca silvicola (C.L. Koch, 1834)

MATERIAL. $1 \sigma^{7}$ (PSU-960), [2], $700 \mathrm{~m}$ a.s.1., mountain taiga, 14.VII.1996, AIE.

DISTRIBUTION. Trans-Palaearctic boreal range.

\section{LINYPHIIDAE} 1875)

Agnyphantes expunctus (O. Pickard-Cambridge,

MATERIAL. 1 ơ (PSU-991), [6], $900 \mathrm{~m}$ a.s.1., tundra, 07.VII.1996, AIE; 1 क (PSU-4790), [3], Pinus-Picea forest, 9.VII.2004, OAP; 1 フ (PSU-4790), [4]: Kolpak Mt., no habitat data, 1.VII.2003, OAP

DISTRIBUTION. Trans-Palaearctic temperate range.

Agyneta conigera (O. Pickard-Cambridge, 1863)

MATERIAL. $1 \mathrm{O}^{7}$ (PSU-911), [2], $700 \mathrm{~m}$ a.s.l., mountain taiga, 12.VII.1996, AIE.

DISTRIBUTION. Trans-EuroSiberian temperate range.

Agyneta gulosa (L. Koch, 1869)

MATERIAL. $10^{7}$ (IPAE), [2], $900 \mathrm{~m}$ a.s.l., tundra, 7.VII.1996, AIE; $8 \sigma^{7} \sigma^{7},[2], 1200-1300 \mathrm{~m}$ a.s.l., Mountain meadow, 10.VII.1997, AIE; $1 \mathrm{O}^{7}$ (PSU-2736), [4]: Bugor Mt., tundra, 11.VIII.2001, AIE

DISTRIBUTION. Trans-Palaearctic boreo-mountain range.

Agyneta subtilis (O. Pickard-Cambridge, 1863)

MATERIAL. $3 \mathrm{O}^{7} \mathrm{O}^{7}, 1$ + (PSU-912), [2], $700 \mathrm{~m}$ a.s.l., mountain taiga, 14.VII.1996, AIE.
DISTRIBUTION. West-Palaearctic temperate range.

Allomengea scopigera (Grube, 1859)

MATERIAL. 1 ○ึ, 3 우 (PSU-2737), [4]: Bugor Mt., mountain taiga, 14.VIII.2001, AIE.

DISTRIBUTION. Circum-Holarctic temperate range.

Bathyphantes setiger F.O. Pickard-Cambridge, 1894 MATERIAL. $10^{7}$ (PSU-917), [2], mountain tundra, 18.VI.1981, O.N. Fileva.

Esyunin [2006]: [2].

DISTRIBUTION. Trans-EuroSiberian temperate range.

Bolyphantes punctulatus (Holm, 1939)

MATERIAL. $1 \sigma^{7}$ (IPAE), [1]: Tylaiskiy Kamen' Mt., grasslichen-moss tundra, 25-28.VII.2011, YEM.

DISTRIBUTION. NW European-Trans-Siberian hypoarcto-mountain range.

Centromerus arcanus (O. Pickard-Cambridge, 1873)

MATERIAL. $1 \sigma^{7}, 2$ 우 (PSU-947), [2], $700 \mathrm{~m}$ a.s.l., mountain taiga, 14.VII.1996, AIE; 1 ㅇ (IPAE), [4]: Bugor Mt., subalpine belt, no habitat data, 14.VIII.2001, AIE

DISTRIBUTION. Euro-West Siberian temperate range.

Cnephalocotes obscurus (Blackwall, 1834)

MATERIAL. 1 + (PSU-4822), [2], Betula-Picea forest, 7.VI. 2003, OAP.

DISTRIBUTION. Circum-Holarctic temperate range.

Diplocephalus picinus (Blackwall, 1841)

MATERIAL. 1 O (PSU-4827), [3], Betula-Picea forest, 7.VI.2003, OAP.

DISTRIBUTION. West-Palaearctic temperate range. 1879)

Entelecara congenera (O. Pickard-Cambridge,

MATERIAL. 1 ○ (PSU-957), [1]: Konzhakovskiy Kamen' Mt., $900 \mathrm{~m}$ a.s.l., grass-lichen-moss tundra, 7.VII.1996, AIE.

Esyunin [2006]: [1].

DISTRIBUTION. West-Central Palaearctic temperate range.

Gonatium rubellum (Blackwall, 1841)

MATERIAL. 3 우 (IPAE), [1]: Tylaiskiy Kamen' Mt., grasslichen-moss tundra, 25-28.VII.2011, YEM.

DISTRIBUTION. Trans-Palaearctic temperate range.

Gonatium rubens (Blackwall, 1833)

MATERIAL. 1 (IPAE), [1]: Garevaya Mt., Vaccinium-grass tundra with dwarf Betula stand, 23-27.VII.2011, YEM; $20^{7} 0^{7}$, 4 아 (IPAE), [2], 1200-1300 m a.s.l., Mountain meadow, 10.VII. 1997, AIE; 1 + (IPAE), [2], tundra, 18.VI.1981, AIE; $1 \mathrm{O}^{7}$ (PSU4839), [3], bank of Kos'va River, 21.VII.2004, OAP

DISTRIBUTION. Trans-Palaearctic temperate range.

Gongylidiellum latebricola (O. Pickard-Cambridge, 1871)

MATERIAL. 1 (PSU-4805), [3], edge of mixed forest, 2.VII.2004, OAP.

DISTRIBUTION. West-Palaearctic nemoral range. 
Helophora insignis (Blackwall, 1841) OAP.

MATERIAL. $1 \mathrm{O}^{\top}$ (PSU-4802), [2], no habitat data, VIII.2005,

DISTRIBUTION. Circum-Holarctic temperate range.

Hypselistes jacksoni (O. Pickard-Cambridge, 1902)

MATERIAL. 2 우 (PSU-4803), [3], edge of coniferous forest, 10.VII.2003, OAP.

DISTRIBUTION. Subcircum-Holarctic temperate range.

Kaestneria dorsalis (Wider, 1834)

MATERIAL. 2 +P (PSU-4789), [3], no habitat data, 23.VI.2003, OAP.

DISTRIBUTION. West-Palaearctic temperate range.

Kaestneria pullata (O. Pickard-Cambridge, 1863) OAP.

MATERIAL. 1 + (PSU-4831), [3], mixed forest, 11.VII.2004,

DISTRIBUTION. Circum-Holarctic temperate range.

Maso sundevalli (Westring, 1851)

MATERIAL. 1 (PSU-4846), [3], mixed forest, 10.VII.2004, OAP.

DISTRIBUTION. Circum-Holarctic temperate range.

Mecynargus monticola (Holm, 1943)

MATERIAL. $1 \sigma^{7}$ (IPAE), [2], 1200-1300 m a.s.l., mountain meadow, 10.VII.1997, AIE; 2 O $^{7}$, (PSU-993), [2], tundra, 18.VI.1981, O.N. Fileva.

Esyunin [2006]: [2].

DISTRIBUTION. EuroSiberian-West Nearctic hypoarcto-boreal range.

Microlinyphia pusilla (Sundevall, 1830)

MATERIAL. $10^{7}, 4$ 우 (PSU-4834), [3], edge of mixed forest, 17.VII.2003, OAP

DISTRIBUTION. Circum-Holarctic temperate range.

Microneta viaria (Blackwall, 1841)

MATERIAL. $2 \bigcirc^{7} \sigma^{7}, 2$ 우 (PSU-4851), [3], multiherbaceous meadow, 17.VI.2003, OAP; 1 ㅇ (PSU-2740), [4]: Bugor Mt., subalpine belt, no habitat data, 14.VIII.2001, AIE.

DISTRIBUTION. Circum-Holarctic temperate range.

Minyriolus pusillus (Wider, 1834)

MATERIAL. $1 \sigma^{7}, 1$ ㅇ (PSU-4835), [4]: Kolpak Mt., forest, 19.VII.2004, OAP

DISTRIBUTION. Trans-EuroSiberian boreal range.

Neriene montana (Clerck, 1757)

MATERIAL. 1 (PSU-4817), [3], no habitat data, 17.VII.2003, OAP.

DISTRIBUTION. Trans-Palaearctic temperate range.

Obscuriphantes obscurus (Blackwall, 1841)

MATERIAL. $10^{7}, 2$ 우 (PSU-4823), [3], heather, 01.VII.2004; OAP.

DISTRIBUTION. West-Palaearctic boreo-mountain range.

Oreoneta leviceps (L. Koch, 1879)

MATERIAL. 2 우 (IPAE), [1]: Tylaiskiy Kamen' Mt., grasslichen-moss tundra, 25-28.VII.2011, YEM; 1 (IPAE), [1]: Konzhakovskiy Kamen' Mt., grass-lichen-moss tundra, 1-3.VIII.2011, YEM.
REMARKS. The present locality is the southernmost one in the Urals; hitherto known from NW Komi Republic [Koponen et al., 1998: as Hilaira; Tanasevitch \& Koponen, 2007] and Sob River, the Polar Urals [Tanasevitch, 1985: as Hilaira; Saaristo \& Marusik, 2004; Tanasevitch et al., 2009].

DISTRIBUTION. NE European-Trans-Nearctic arctic range.

Oreonetides vaginatus (Thorell, 1872)

MATERIAL. 1 \% (PSU-4850), [2], no habitat data, VIII.2005, OAP; 2 우 (PSU-2741), [4]: Bugor Mt., subalpine belt, no habitat data, 14.VIII.2001, AIE.

DISTRIBUTION. Circum-Holarctic temperate range.

Panamomops tauricornis (Simon, 1881)

MATERIAL. 1 ㅇ (PSU-1013), [3], $900 \mathrm{~m}$ a.s.l., tundra, 7.VII.1996, AIE.

Esyunin [2006]: [3].

DISTRIBUTION. Trans-EuroSiberian boreo-mountain range.

Pelecopsis mengei (Simon, 1884)

MATERIAL. $1 \sigma^{\text {T⿱ }}, 1$ क (IPAE), [1]: Tylaiskiy Kamen' Mt., grass-lichen-moss tundra, 25-28.VII.2011, YEM; $1 \mathrm{O}^{\top}$ (PSU-4812), [3], Pinus-Picea forest, 18.VII.2004, OAP.

DISTRIBUTION. Circum-Holarctic temperate range.

Perregrinus deformis (Tanasevitch, 1982)

MATERIAL. 2 +o (PSU-1015), [2], 1500 m a.s.1., Carex-moss tundra, 9.VII.1996, AIE.

Esyunin [2006]: [2].

DISTRIBUTION. NEEuropean-Trans-Nearctic hypoarcto-boreal range.

Savignya frontata Blackwall, 1833

MATERIAL. 1 o (PSU-4794), [3], Pinus-Picea forest, 13.VII.2004, OAP.

DISTRIBUTION. Euro-West Siberia temperate range.

Tenuiphantes alacris (Blackwall, 1853)

MATERIAL. 1 (PSU-989), [2], $700 \mathrm{~m}$ a.s.1., mountain taiga, 14.VII.1996, AIE; 2 우 (PSU-4778), [3], mixed forest, 19.VI.2004, OAP.

DISTRIBUTION. Trans-Palaearctic temperate range.

Tenuiphantes nigriventris (C.L. Koch, 1879)

MATERIAL. 2 우 (PSU-1017), [2], $700 \mathrm{~m}$ a.s.1., mountain taiga, 10.VII.1996, AIE; $2 \mathrm{O}^{7} \mathrm{O}^{7}, 4$ 우 (PSU-4819), [3], mixed forest and heather, 19.VI \& 1.VII.2004, OAP; $1 O^{7}$ (PSU-1017), [4]: Bugor Mt., subalpine belt, no habitat data, 14.VIII.2001, AIE.

DISTRIBUTION. NW European-Trans-Siberian boreal range.

Tenuiphantes tenebricola (Wider, 1834)

MATERIAL. $1 \sigma^{7}$ (PSU-1028), [2], $700 \mathrm{~m}$ a.s.1., mountain taiga, 14.VII.1996, AIE; 1 O7, [2], $900 \mathrm{~m}$ a.s.1., tundra, 10.VII.1996, AIE.

DISTRIBUTION. West-Palaearctic temperate range. 1873)

Walckenaeria karpinskii (O. Pickard.-Cambridge,

MATERIAL. 1 (IPAE), [1]: Tylaiskiy Kamen’ Mt., grasslichen-moss tundra, 25-28.VII.2011, YEM. 
DISTRIBUTION. Circum-Holarctic arcto-boreal range.

Walckenaeria vigilax (Blackwall, 1853) OAP.

MATERIAL. $1 \mathrm{O}^{\mathrm{T}}$ (PSU-4852), [3], mixed forest, 23.VI.2004,

DISTRIBUTION. West Palaearctic-West Nearctic disjunctive temperate range.

Zornella cultrigera (L. Koch, 1879)

MATERIAL. 1 \% (PSU-1042), [2], $700 \mathrm{~m}$ a.s.1., Mountain taiga, 14.VII.1996, AIE; 1 O$^{7}$ (PSU-1042), [4]: Bugor Mt., mountain taiga, 14.VIII.2001, AIE.

DISTRIBUTION. Euro-Middle Siberian boreal range.

\section{LIOCRANIDAE}

Agroeca brunnea (Blackwall, 1833)

MATERIAL. 1 + (PSU-4784), [3], Betula-Picea forest, 28.VI.2003, OAP.

DISTRIBUTION. Trans-Palaearctic temperate range.

\section{LYCOSIDAE}

\section{Acantholycosa lignaria (Clerck, 1757)}

Kharitonov [1926: as Lycosa lignaria]: south slope TylaiskoKonzhakovskiy Mt. Ridge.

DISTRIBUTION. Trans-EuroSiberian temperate range.

Acantholycosa norvegica (Thorell, 1872) $3(+\circ)$.

Lycosa (Acantholycosa) sudetica: Kharitonov, 1926: 54, Fig.

MATERIAL. 2 +,+ 1 subadult $\sigma^{\top}$ (IPAE), [1]: Garevaya Mt., Vaccinium-grass tundra with dwarf Betula stand, 23-27.VII.2011, YEM; $6 \sigma^{7} \sigma^{7}, 1$ subadult $\sigma^{7}, 2$ juv. (IPAE), [1]: Tylaiskiy Kamen' Mt., grass-lichen-moss tundra, 25-28.VII.2011, YEM; $1 \sigma^{\top}, 1$ \% (IPAE), [1]: Serebryanskiy Kamen' Mt., 1200 m a.s.1., dwarf Betula stand, 12.VII.1996, AIE; $2 \sigma^{7} \sigma^{7}$ (IPAE), [2], 1200-1300 m a.s.1., mountain meadow, 9.VII.1997, AIE; 1 \% (PSU-4820), [2], stone streams (=kurumy), 27.VI.2003, OAP.

Kharitonov [1926: as Lycosa sudetica L. Koch, 1875]: the top of Tylaisko-Konzhakovskiy Mt. Ridge.

DISTRIBUTION. Trans-EuroSiberian disjunctive boreo-mountain range.

Alopecosa taeniata (C.L. Koch, 1835)

MATERIAL. $4 \sigma^{\top} \sigma^{\top}, 2$ OO (IPAE), [2], $900 \mathrm{~m}$ a.s.1., tundra, 710.VII.1996, 8.VII.1997, AIE; 7 O $^{\top}, 3$ ○ी (PSU-4847), [4]: Kolpak Mt., tundra, VI.2004, OAP.

Kharitonov [1926: as Tarentula aculeata (Clerck, 1757) - 1 o misidentification]: the south slope of Tylaisko-Konzhakovskiy Mt. Ridge.

DISTRIBUTION. West-Palaearctic temperate range.

Pardosa agrestis (Westring, 1861)

MATERIAL. $1 \sigma^{\top}$, [1]: Tylaiskiy Kamen' Mt., grass-lichenmoss tundra, 25-28.VII.2011, YEM.

DISTRIBUTION. West-Palaearctic polyzonal range.

Pardosa amentata (Clerck, 1757)

MATERIAL. $1 \overbrace{}^{7}$ (PSU-1004), [2], $700 \mathrm{~m}$, coniferous forest, 14.VII.1996, AIE.

DISTRIBUTION. West-Palaearctic polyzonal range.
Pardosa fulvipes (Collett, 1875)

MATERIAL. 1 (PSU-4795), [3], multiherbaceous meadow, 17.VI.2003, OAP.

DISTRIBUTION. West-Palaearctic temperate range.

Pardosa hyperborea (Thorell, 1872)

MATERIAL. $2 \sigma^{7} \sigma^{7}, 33$ 우, 3 subadult $0^{7} \sigma^{7}, 2$ subadult 우우 (IPAE), [1]: Garevaya Mt., Vaccinium-grass tundra with dwarf Betula stand, 23-27.VII.2011, YEM; 10 Oొ $^{7}, 4$ 우 (PSU-1014), [1]: Serebryanskiy Kamen' Mt., 1200 m a.s.l., dwarf Betula stand, 12.VII.1996, AIE; 2 o $^{7} \sigma^{7}$ (IPAE), [2], $900 \mathrm{~m}$, tundra, 10.VII.1996, AIE; 16 우 (PSU-4800), [4]: Kolpak Mt., tundra, 7.VII.2004, OAP; 4 우 (PSU-2742), [4]: Bugor Mt., tundra, VIII.2001, AIE.

DISTRIBUTION. Subcircum-Holarctic hypoarctoboreal range.

Pardosa lapponica (Thorell, 1872)

MATERIAL. 2 우 (IPAE), [1]: Trapetsiya Mt., grass-mossy tundra with Vaccinium, 3.VIII.2011, YEM.

DISTRIBUTION. EuroSiberian-West Nearctic boreo-mountain range.

Pardosa lasciva (L. Koch, 1879)

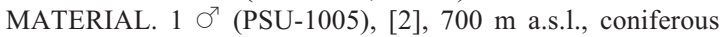
forest, 14.VII.1996, AIE.

DISTRIBUTION. NW European-Trans-Siberian boreo-mountain range.

Pardosa lugubris (Walckenaer, 1802)

MATERIAL. $2 \sigma^{7} \sigma^{7}$ (PSU-1006), [2], $700 \mathrm{~m}$ a.s.1., coniferous forest, 14.VII.1996, AIE; 1 ( (PSU-4809), [2], no habitat data, VIII.2005, OAP; $1 \mathrm{O}^{\top}$ (PSU-4809), [3], edge of mixed forest, 28.VI.2003, OAP.

DISTRIBUTION. West-Palaearctic temperate range.

Pardosa palustris (Linnaeus, 1758)

MATERIAL. $10^{7}$ (IPAE), [2], 900 m a.s.1., tundra, 10.VII.1996, AIE; 2 우 (PSU-4825), [3], multiherbaceous meadow, 20.VI.2004, OAP.

DISTRIBUTION. Trans-Palaearctic-West Nearctic temperate range.

Pardosa pullata (Clerck, 1757)

MATERIAL. 1 + (PSU-4832), [3], edge of mixed forest, 23.VI.2003, OAP.

DISTRIBUTION. West-Central Palaearctic temperate range.

Pardosa riparia (C.L. Koch, 1833)

MATERIAL. $10^{7}$ (IPAE), [1]: Garevaya Mt., Vaccinium-grass tundra with dwarf Betula stand, 23-27.VII.2011, YEM; $30^{7} \sigma^{7}$, 1 ( (IPAE), [1]: Tylaiskiy Kamen' Mt., grass-lichen-moss tundra, 25-28.VII.2011, YEM; 3 O $^{7} \sigma^{7}, 1$ + (IPAE), [1]: Trapetsiya Mt., grass-mossy tundra with Vaccinium, 2-3.VIII.2011, YEM; $10^{7}$ (IPAE), [1]: Serebryanskiy Kamen' Mt., 1200 m a.s.l., dwarf Betula stand, 12.VII.1996, AIE; $1 \sigma^{7}$ (IPAE), [2], $700 \mathrm{~m}$, coniferous forest, 14.VII.1996, AIE; $2 \sigma^{7} \sigma^{7}$ (IPAE), [2], $900 \mathrm{~m}$ a.s.l., tundra, 10.VII.1996, AIE; 5 O$^{7} \sigma^{7}, 1$ \% (PSU-4838), [3], edge of mixed forest, 28.VI.2003, OAP; $10^{7}, 9$ oq (PSU-4838), [4]: Kolpak Mt., mountain meadow, 11.VI.2004, OAP

DISTRIBUTION. Trans-Palaearctic temperate range.

Pardosa schenkeli Lessert, 1904

Lycosa schenkeli: Kharitonov, 1926: 56, Fig. 4 (†).

MATERIAL. $7 \sigma^{7} \sigma^{7}, 19$ (PSU-1007), [2], $900 \mathrm{~m}$ a.s.l., tundra, 7-10.VII.1996, AIE; 1 ㅇ (PSU-4840), [4]: Kolpak Mt., tundra, 10.VII.2004, OAP. 
Kharitonov [1926: as Lycosa schenkeli (Lesset, 1904)]: [2].

DISTRIBUTION. Trans-EuroSiberian boreo-mountain range.

Pardosa sphagnicola (F. Dahl, 1908)

MATERIAL. 1 ○, 1 (PSU-4843), [3], multiherbaceous meadow, 11.VI.2004, OAP.

DISTRIBUTION. Euro-West Siberian temperate range.

Pirata hygrophilus Thorell, 1872

MATERIAL. $1 \sigma^{7}$ (PSU-4799), [4]: Kolpak Mt., swamped moss slope, 4.VII.2004, OAP.

DISTRIBUTION. West-Central-Palaearctic temperate range.

Xerolycosa nemoralis (Westring, 1861)

MATERIAL. $1 \sigma^{7}$ (PSU-1037), [2], $900 \mathrm{~m}$ a.s.1., tundra, 10.VII.1996, AIE; 1 O $^{7}$ (PSU-4818), [3], meadow, 27.VI.2003, OAP; 3 OO (PSU-4818), [4]: Kolpak Mt., tundra, 6.VII.2004, OAP.

DISTRIBUTION. Trans-Palaearctic temperate range.

\section{METIDAE}

Metellina mengei (Blackwall, 1869)

MATERIAL. $20^{7} \sigma^{7}, 3$ 우 (PSU-4811), [3], edge of mixed forest, 17.VI.2003, OAP

DISTRIBUTION. West-Palaearctic temperate range.

MIMETIDAE

Ero furcata (Villers, 1789)

MATERIAL. 1 + (PSU-4796), [3], Betula-Picea forest, 17.VI.2003, OAP.

DISTRIBUTION. Trans-Palaearctic temperate range.

\section{PHILODROMIDAE}

Philodromus cespitum (Walckenaer, 1802)

MATERIAL. 1 \& (PSU-4787), [3], multiherbaceous meadow, 17.VI.2003, OAP

DISTRIBUTION. Circum-Holarctic polyzonal range.

Philodromus margaritatus (Clerck, 1757)

MATERIAL. 1 O $^{7}$ (PSU-1552), [1]: Tylaiskiy Kamen’ Mt., no habitat data, 27.VII.1925, A. Tauson.

DISTRIBUTION. Trans-Palaearctic temperate range.

Thanatus striatus C.L. Koch, 1845

MATERIAL. 1 (PSU-4844), [3], meadow bog on river bank, 7.VI.2004, OAP

DISTRIBUTION. Circum-Holarctic polyzonal range.

Tibellus oblongus (Walckenaer, 1802)

MATERIAL. $1 \mathrm{O}^{\text {T }}$ (PSU-1018), [2], $900 \mathrm{~m}$ a.s.l., tundra, 10.VII.1996, AIE: 1 O$^{7}, 3$ 우 (PSU-4821), [3], edge of mixed forest, 17.VI.2003, OAP.

DISTRIBUTION. Circum-Holarctic polyzonal range.

\section{PISAURIDAE}

Dolomedes fimbriatus (Clerck, 1757)

MATERIAL. $10^{7}$ (PSU-4792), [3], meadow, 28.VI.2003, OAP. Kharitonov [1926]: [2], bank of lake.

DISTRIBUTION. West-Central-Palaearctic temperate range.
SALTICIDAE

Chalcoscirtus alpicola (L. Koch, 1876)

MATERIAL. $10^{7}$ (IPAE), [1]: Garevaya Mt., Vaccinium-grass tundra with dwarf Betula stand, 23-27.VII.2011, YEM

DISTRIBUTION. Circum-Holarctic hypoarctoboreo-montane range.

Evarcha arcuata (Clerck, 1757)

MATERIAL. 1 \& (PSU-4780), [4]: Kolpak Mt., stone streams (=kurumy), 11.VII.2004, OAP

DISTRIBUTION. Trans-Palaearctic temperate range.

Evarcha falcata (Clerck, 1757)

MATERIAL. 4 O $^{7} \mathrm{O}^{7}, 1$ ㅇ (PSU-4790), [3], edge of mixed forest, 17.VI.2003, OAP

DISTRIBUTION. West-Central-Palaearctic temperate range.

Heliophanus camtschadalicus Kulczyński, 1885

MATERIAL. $1 \sigma^{7}$ (PSU-966), [2], $900 \mathrm{~m}$ a.s.l., stone streams (=kurumy), 8.VII.1996, AIE; 2 +ᄋ (PSU-4785), [3], edge of mixed forest, 17.VI.2003, OAP.

DISTRIBUTION. Trans-EuroSiberian boreal range.

Neon reticulatus (Blackwall, 1853)

MATERIAL. $1 \sigma^{\top}$ (PSU-4837), [3], Betula-Picea forest, 17.VI. 2003, OAP

DISTRIBUTION. Trans-Palaearctic-West Nearctic temperate range.

Synageles venator (Lucas, 1836)

MATERIAL. $1 \sigma^{7}$ (PSU-2743), [4]: Semichelovechiya Mt., tundra, 6.VIII.2001, AIE.

DISTRIBUTION. Trans-Palaearctic temperate range.

Talavera petrensis (C.L. Koch, 1837)

MATERIAL. 1 + (PSU-4826), [3], no habitat data, 21.VI.2004, OAP.

REMARKS. The present locality is the northernmost one in the Urals; hitherto known from Perm, Orenburg and Chelyabinsk Areas [Esyunin \& Efimik, 1996; Logunov \& Marusik, 2000].

DISTRIBUTION. West-Central-Palaearctic nemoral range.

\section{TETRAGNATHIDAE}

Tetragnatha extensa (Linnaeus, 1758)

MATERIAL. 1 ○ (PSU-1030), [1]: Serebryanskiy Kamen' Mt., $1200 \mathrm{~m}$ a.s.1., dwarf Betula stand, 12.VII.1996, AIE; 1 우 (PSU-1029), [2], mountain taiga, 13.VII.1996, AIE.

Kharitonov [1926]: [3], banks of river and lake.

DISTRIBUTION. Circum-Holarctic polyzonal range.

Tetragnatha pinicola $\mathrm{L}$. Koch, 1870

MATERIAL. 1 ㅇ (PSU-1033), [2], $900 \mathrm{~m}$ a.s.1., tundra, 7.VII.1996, AIE; $2 O^{\top} \mathrm{O}^{\top}, 2$ 우 (PSU-4828), [3], meadow, 1.VIII.2004, OAP.

DISTRIBUTION. Trans-Palaearctic temperate range.

\section{THERIDIIDAE}

Crustulina guttata (Wider, 1834) OAP.

MATERIAL. 1 (PSU-4798), [3], no habitat data, 10.VIII.2004, 
DISTRIBUTION. Trans-Palaearctic temperate range.

Euryopis flavomaculata (C.L. Koch, 1836) OAP.

MATERIAL. $1 \mathrm{O}^{7}$ (PSU-4793), [3], no habitat data, 7.VI.2004,

DISTRIBUTION. Euro-Siberian temperate range.

Ohlertidion ohlerti (Thorell, 1870)

MATERIAL. $1 \sigma^{7}$ (PSU-4824), [4]: south slope of Kolpak Mt., no habitat data, 12.VII.2003, OAP.

DISTRIBUTION. Circum-Holarctic boreo-mountain range.

Phylloneta impressa (L. Koch, 1881)

MATERIAL. $1 \mathrm{O}^{7}$ (PSU-4801), [3], multiherbaceous meadow, 19.VI.2003, OAP.

DISTRIBUTION. Subcircum-Holarctic polyzonal range.

Phylloneta sisyphia (Clerck, 1757)

MATERIAL. $2 \mathrm{O}^{7} \sigma^{7}$ (PSU-4842), [2], stone streams (=kurumy), 27.VI.2003, OAP.

DISTRIBUTION. West-Central-Palaearctic polyzonal range.

Robertus lividus (Blackwall, 1836)

MATERIAL. $10^{7}$ (PSU-1023), [2], $700 \mathrm{~m}$ a.s.1., coniferous forest, 14.VII.1996, AIE; $10^{7}, 2$ O+क (PSU-4807), [2], no habitat data, VIII.2005, OAP; $2 \bigcirc^{7} \sigma^{7}, 2$ 우 (PSU-1023), [4]: Bugor Mt. mountain forest, VIII.2001, AIE.

DISTRIBUTION. Trans-Palaearctic-West Nearctic temperate range.

Steatoda albomaculata (DeGeer, 1778)

MATERIAL. 6 O$^{7} \sigma^{7}, 4$ 우 (PSU-4779), [3], river bank, 4.VII.2003, OAP.

DISTRIBUTION. Circum-Holarctic polyzonal range.

Thymoites bellissimus (L. Koch, 1879)

MATERIAL. 1 (PSU-2744), [4]: Bugor Mt., mountain taiga, 14.VIII.2001, AIE

DISTRIBUTION. NW European-Trans-Siberian boreal range.

\section{THOMISIDAE}

Misumena vatia (Clerck, 1757)

MATERIAL. 1 juv. (IPAE), [2]: slope of Kos'vinskiy Kamen' Mt., mountain taiga 13.VII.1996, AIE.

DISTRIBUTION. Circum-Holarctic polyzonal range.

Ozyptila arctica Kulczyński, 1908

MATERIAL. $10^{\text {T }}, 2$ 우 (IPAE), [1]: Garevaya Mt., Vaccinium-grass tundra with dwarf Betula stands, 23-27.VII.2011, YEM

DISTRIBUTION. EuroSiberian-West Nearctic hypoarcto-mountain range.

Ozyptila trux (Blackwall, 1846)

MATERIAL. $1 \sigma^{7}$ (PSU-1052), [2]: $700 \mathrm{~m}$ a.s.1., coniferous forest, 14.VII.1996, AIE; 1 ㅇ (PSU-4848), [3], multiherbaceous meadow, 4.VI.2004, OAP; 1 + (PSU-1052), [4]: Bugor Mt., Mountain taiga, 11.VIII.2001, AIE.

DISTRIBUTION. Trans-Palaearctic temperate range.
Xysticus austrosibiricus Logunov et Marusik, 2001

MATERIAL. $1 \sigma^{7}$ (IPAE), [2], $900 \mathrm{~m}$ a.s.1., tundra, 10.VII.1996, AIE; 8 O $^{7} \sigma^{7}, 1$ (IPAE), [2], 1200-1300 m a.s.1., mountain meadow, 9-10.VII.1997, AIE.

DISTRIBUTION. West-Middle Siberian boreomountain range.

Xysticus bifasciatus C.L. Koch, 1837

MATERIAL. $60^{7} \sigma^{7}$ (PSU-4782), [3], multiherbaceous meadow, 20.VII.2003, OAP.

DISTRIBUTION. West-Central Palaearctic temperate range.

Xysticus bonneti Denis, 1937

MATERIAL. 1 (IPAE), [1]: Garevaya Mt., Vaccinium-grass tundra with dwarf Betula stands, 23-27.VII.2011, YEM; 1 (PSU4783), [2], stone streams (=kurumy), 23.VI.2003, OAP; 5 O $^{7}$ (PSU-4783), [4]: Kolpak Mt., stone streams (=kurumy), 11.VII.2004, OAP; 1 ㅇ (PSU-2745), [4]: Semichelovechiya Mt., tundra, 6.VIII.2001, AIE

DISTRIBUTION. Euro-Middle Siberian mountain disjunction range.

Xysticus cristatus (Clerck, 1757)

MATERIAL. $2 \bigcirc^{7} \Im^{7}, 2$ ㅇ (PSU-4853), [3], edge of mixed forest, 17.VI.2003, OAP

Kharitonov [1926: as Xysticus pini (Hahn, 1831) - 1 \%, misidentification]: [1], Serebryanskiy Kamen’ Mt.

DISTRIBUTION. Trans-Palaearctic temperate range.

Xysticus luctuosus (Blackwall, 1836)

MATERIAL. $60^{7} 0^{7}, 1$ (PSU-4808), [3], multiherbaceous meadow, 20.VII.2003, OAP; 3 Oొ $^{7}$ (PSU-4808), [4]: Kolpak Mt., tundra, 11.VI.2004, OAP

DISTRIBUTION. Circum-Holarctic temperate range.

Xysticus ulmi (Hahn, 1832) OAP

MATERIAL. $10^{\text {T’ }}, 1$ (PSU-4849), [3], meadow, 20.VII.2003,

DISTRIBUTION. West-Central Palaearctic temperate range.

\section{ZORIDAE}

Zora nemoralis (Blackwall, 1861)

MATERIAL. $1 \sigma^{7}$ (PSU-1041), [2], $900 \mathrm{~m}$ a.s.1., tundra, 10.VII.1996, AIE.

DISTRIBUTION. West-Central-Palaearctic temperate range.

Although the local spider fauna of the Basegi Ridge (Middle Urals; 262 species [Esyunin, Efimik, 1996]) is well studied, the information on spiders of the vicinity of Kytlym remains inadequate. A real diversity of this spider fauna seems to be not less than 250 species.

ACKNOWLEDGEMENTS. This research project was funded by the Russian Foundation for Basic Research (grants \#\# 09-04-01365, 12-04-01548) and by the grant to support the leading scientific schools from the President of the Russian Federation. In 2011, the spider collection was made by Yu.E. Mikhailov within the frames of 'GLORIA Invertebrate monitoring' supported by the Austrian Academy of 
Sciences (grant ÖAW 621/11). The English of the final draft was kindly checked by D.V. Logunov, Manchester, UK.

\section{References}

Esyunin S.L. 2006. Remarks on the Ural spider fauna (Arachnida: Aranei). 11. New faunistic records // Arthropoda Selecta. Vol.14 (for 2005). No.4. P.329-337.

Esyunin S.L., Efimik V.E. 1996. Catalogue of the spiders (Arachnida, Aranei) of the Urals. Moscow: KMK Scientific Press Ltd. 229 p.

Esyunin S.L., Efimik V.E. 1997a. [Some results of the Urals spider fauna study] // Vestnik Perm. Univ. No.3. P.122-127 [in Russian]

Esyunin S.L., Efimik V.E. 1997b. [The spider fauna of Urals: history of investigation and some results] // Uspekhi entomologii na Urale. Ekaterinburg. P.118-121 [in Russian].

Esyunin S.L., Ermakov À.I. 2012. [Spider fauna (Aranei) of the Denezhkin Kamen' Reserve, Ekaterinburg (= Sverdlovskaya) Area] // Vestnik Perm. Universiteta. Ser. Biology. No.2. P.2235 [in Russian].

Esyunin S.L., Marusik Yu.M. 2011. [An attempt of the classification of distributional patterns of the Ural's spiders] // Vestnik Perm. Univ. Biology. No.1. P.32-36 [in Russian].

Kharitonov D.E. 1926. [Materials on the spider fauna of the Verkhnyaya Tura Urals] // Izv. biolog. Nauch.-Issl. instituta pri Perm. gos. un-te. Vol.5. No.2. P.49-60 [in Russian].

Kharitonov D.E. 1936. [Spiders of the Denezhkin Kamen' Mt. and its vicinities (North Urals)] // Uchenye zapiski Perm. gos. unta. Vol.2. No.3. P.15-35 [in Russian].
Koponen S., Marusik Yu.M., Tanasevitch A.V. 1998. New data on the spider fauna of the Polar Urals (Aranei) // Arthropoda Selecta. Vol.6 (for 1997). No.3/4. P.109-119.

Moiseev P.A., Bartysh A.A., Nagimov Z.Y. 2010. Climate changes and tree stand dynamics at the upper limit of their growth in the North Ural mountains // Russian Journal of Ecology. Vol.41. No.6. P.486-497.

Saaristo M.I., Marusik Yu.M. 2004. Revision of the Holarctic spider genus Oreoneta Kulczyński, 1894 (Arachnida: Aranei: Linyphiidae) // Arthropoda Selecta. Vol.12 (for 2003). No.34. P.207-249.

Tanasevitch A.V. 1985. [To the study of spiders (Aranei) of the Polar Urals] // Fauna i ekologiya paukov SSSR. Trudy Zool. Inst. AN SSSR. Leningrad: Nauka. Vol.139. P.52-62 [in Russian].

Tanasevitch A.V., Koponen S. 2007. Spiders (Aranei) of the southern tundra in the Russian Plain // Arthropoda Selecta. Vol.15 (for 2006). No.4. P.295-345

Tanasevitch A.V., Koponen S., Kaarlejarvi E. 2009. Small collection of spiders (Aranei) from the Polar Urals and Yamal Peninsula, Russia // Arthropoda Selecta. Vol.17 (for 2008). No.3-4. P.185-190.

Tuneva T.K. 2007. Review of the family Gnaphosidae on the Ural fauna (Aranei), 5. Genera Micaria Westring, 1851 and Arboricaria Bosmans, 2000 // Arthropoda Selecta. Vol.15 (for 2006). No.3. P.229-250.

Responsible editor D.V. Logunov 